\section{The penetration of methanol into bovine cardiac and hepatic tissues is faster than ethanol and formalin}

\author{
Michelle Steicke, ${ }^{1}$ Guang Yang, \\ Tam Nguyen Dinh, ${ }^{1}$ \\ Matthew Dunster-Jones, ${ }^{1}$ \\ Owen Sargisson, ${ }^{1}$ Farah Ahmady, \\ Jonathan Golledge, ${ }^{3,4}$ Yutang Wang \\ ${ }^{1}$ School of Applied and Biomedical \\ Science, Federation University \\ Australia, Ballarat, Australia \\ ${ }^{2}$ Department of Geriatric Cardiology, \\ Qianfoshan Hospital Affiliated to \\ Shandong University, Jinan, Shandong \\ Province, China \\ ${ }^{3}$ The Vascular Biology Unit, Queensland \\ Research Centre for Peripheral Vascular \\ Disease, College of Medicine and \\ Dentistry, James Cook University, \\ Townsville, Australia \\ ${ }^{4}$ Department of Vascular and \\ Endovascular Surgery, The Townsville \\ Hospital, Australia
}

\begin{abstract}
Methanol, ethanol and formalin are commonly used as fixatives to preserve biological tissues from decay in the preparation of histological sections. Fixation of the inner layers of the tissue depends on the ability of the fixative to diffuse into the tissue. It is unknown whether methanol penetrates tissues at similar rates to other fixatives. This study aimed to compare the penetration rates of methanol, ethanol and formalin into bovine heart and liver tissues. The penetration distance and tissue shrinkage or expansion were measured by analysing the digital images of tissue before and after immersion in different fixatives for 1, 2,6 or $10 \mathrm{~h}$. Data were analysed using two-way ANOVA, followed by Bonferroni's post-hoc test. The penetration distance of methanol was significantly greater in both heart and liver tissues compared with that of ethanol $(\mathrm{N}=4$, $\mathrm{P}<0.001)$. Methanol or ethanol immersion led to similar shrinkage of both tissues $(\mathrm{P}>0.05)$. The penetration rate of formalin was similar to that of ethanol in both tissues however it was significantly slower than methanol $(\mathrm{N}=4, \mathrm{P}<0.005$ in the heart; $\mathrm{P}<0.001$ in the liver). The mean penetration coefficients of methanol, formalin and ethanol in the heart tissue were 2.609, 1.994 and 1.801, respectively, and 3.012, 2.153 and 2.113 , respectively, in the liver tissue. The
\end{abstract}

penetration coefficient of methanol was significantly greater than that of ethanol or formalin in both tissues $(\mathrm{P}<0.001$ for each comparison). In conclusion, methanol penetrates tissue significantly faster than ethanol and formalin.

\section{Introduction}

A key step in the preparation of biological tissues for histology is the prevention of decay by fixation. ${ }^{1}$ Ten percent neutralbuffered formalin (a 4\% buffered formaldehyde solution) has become the preservative of choice with most pathologists due to the resulting excellent preservation of tissue morphology for long periods of time. ${ }^{2}$ However, formalin fixation is sometimes not used for immunohistochemistry since visualisation of certain antigens can be hampered. ${ }^{3,4}$ Formalin is also not favoured when nuclear fixation is needed. ${ }^{5}$ In addition, DNA and RNA extracted from formalin-fixed material can be difficult. ${ }^{6}$ Therefore alternative fixatives are often used when immunohistochemistry for certain antigens or later isolation of DNA and RNA may be needed.

Methanol and ethanol are frequently used alternative tissue fixatives to formalin. They can be used alone ${ }^{7,8}$ or in combination with other fixatives. ${ }^{4-7}$ Combinations of methanol and ethanol fixation, such as the PAXgene fixative, have been reported to outperform formalin in terms of preserving the integrity of $\mathrm{RNA}^{9}$ and $\mathrm{DNA}^{6}{ }^{6}$ while maintaining the ability to visualise antigens during immunohistochemistry and preserving tissue morphology. ${ }^{9}$

Fixation of the inner layers of tissues depends on the ability of the fixative to diffuse into the tissues. Traditionally it has been assumed that the tissue penetration rates of ethanol and methanol are similar, but there has been no evidence reported to support this assumption. ${ }^{10}$ The aim of this study was to compare the penetration rates of methanol, ethanol and formalin into bovine heart and liver tissues. We chose these two tissues to study because they have a relatively homogeneous structure compared with other organs, such as skin and intestine. ${ }^{11}$

\section{Materials and Methods}

\section{Tissue source}

Four fresh bovine hearts and four fresh bovine livers were purchased from a local butcher (Sinclair Meats).
Correspondence: Yutang Wang, School of Applied and Biomedical Science, Federation University Australia, Ballarat, Victoria 3353, Australia

Tel: +61.3.53279394 - Fax: +61.3.53279289. E-mail: Yutang.wang@federation.edu.au

Key words: Ethanol; formalin; methanol; penetration coefficient; penetration rate

Conflict of interest: The authors declare no conflict of interest.

Acknowledgements: We thank Fahima Ahmady for technical support. YW is supported by a grant from the National Health and Medical Research Council (1062671). JG is supported by grants from the National Health and Medical Research Council (1079369, 1079193, 1063476, 1021416, and 1000967) and Queensland Government, and by a Practitioner Fellowship from the National Health and Medical Research Council (1117061).

Received for publication: 8 December 2017 Accepted for publication: 1 February 2018.

This work is licensed under a Creative Commons Attribution-NonCommercial 4.0 International License (CC BY-NC 4.0).

CC Copyright M. Steicke et al., 2018

Licensee PAGEPress, Italy

European Journal of Histochemistry 2018; 62:2880 doi:10.4081/ejh.2018.2880

\section{Tissue fixation}

Three fixatives were used: ethanol (100\%), methanol (100\%) and 10\% neutralbuffered formalin. The tissue from hearts and livers, which was parenchymal in nature and did not include fibrous or adipose tissue, was cut into $2 \times 2 \times 2 \mathrm{~cm}^{3}$ cubes (12 cubes from each heart or each liver). The tissue cubes, with the marked faces pointing up during the whole experiment, were then immersed into $40 \mathrm{~mL}$ of fixative in a $50-\mathrm{mL}$ specimen collection container and were kept in a room with the temperature controlled at $37^{\circ} \mathrm{C}$. The heart and liver cubes were then taken out of the fixative at $1,2,6$ or $10 \mathrm{~h}$.

\section{Tissue shrinkage or expansion}

The top face of the tissue cube was marked with tissue ink (Shandon ${ }^{\mathrm{TM}}$ TissueMarking Dye, ThermoFisher Scientific, Waltham, MA, USA) and then photographed alongside a ruler immediately before fixation using a Sony Xperia Mobile camera (Tokyo, Japan) (Figure 1A). The tissue cubes were immersed in fixatives with the marked face always pointing up. The tissue blocks were then taken out of the fix- 
ative at certain time points (i.e., either 1, 2, 6 or $10 \mathrm{~h}$ ) and the marked faces were imaged again (Figure 1B). The area of the marked face of the tissue before or after fixation was measured using Photoshop software (ver. CC 2017, San Jose, CA, USA). The change in the marked surface area ( $\Delta$ area) was then calculated. The square root of the absolute value of the ratio of $\Delta$ area/area before fixation was then calculated as the shrinkage or expansion coefficient of the tissue. Positive values represent tissue expansion and negative values represent shrinkage.

\section{Penetration distance measurement}

After the marked face of the fixed tissue cube was photographed, the tissue was cut into two halves along the middle plane parallel to the marked face. The freshly cut face was photographed alongside a ruler for the penetration distance measurement (Figure 2A). The discoloration area represented the penetrated area (Figure $2 \mathrm{~B}$ ). ${ }^{7}$ The penetration distance was measured 40 times at locations equally distributed around the perimeter of the cut face using PhotoShop software (ver. CC 2015). The mean of the 40 measurements was calculated as the penetration distance for an individual image.

\section{Normalisation of penetration distance}

The penetration distance was normalised to the individual tissue's shrinkage or expansion. If the tissue had expanded, normalisation was calculated using the following formula: Normalised penetration distance $=$ non-normalised penetration distance / (1+ shrinkage or expansion coefficient). If the tissue had shrunk, normalisation was calculated using the following formula: normalised penetration distance $=$ non-normalised penetration distance / (1shrinkage or expansion coefficient).

\section{Penetration coefficient}

It has been observed that the penetration distance in millimetres $(x)$ of a given fixative depends on the following simple function of the fixation time in hours $(t){ }^{1,8,12}$

$$
x=\mathrm{K} \sqrt{t}
$$

The constant $\mathrm{K}$ is called the penetration coefficient. We plotted the penetration distance in millimetres relative to the square root of the fixation time in hours using GraphPad Prism 6 software (GraphPad Software, Inc., La Jolla, CA, USA) and obtained the penetration coefficient as the slope of the regression line using the linear regression function.

\section{Statistical analysis}

Differences in shrinkage or expansion, penetration distance and penetration coefficient at various time points among the three fixatives were analysed using two-way ANOVA, followed by Bonferroni's post-hoc test.

\section{Results}

\section{Measurement variability}

Variability in measuring area of the marked face

To measure the tissue expansion or
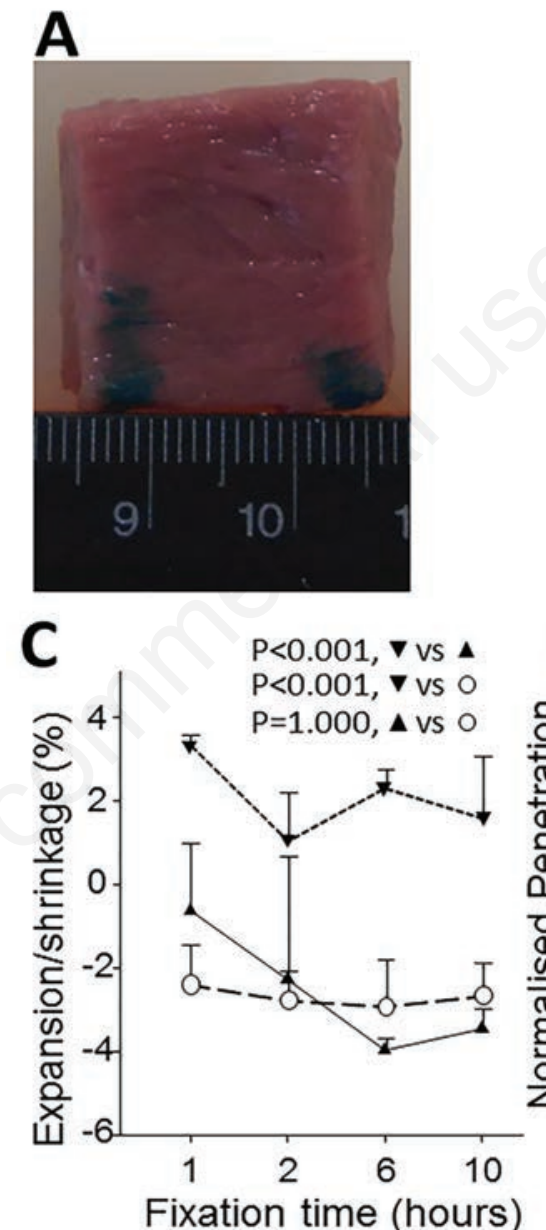

\section{$\Delta$ : ethanol; $\mathbf{v}$ : formalin; o: methanol.}

Figure 1. The normalised penetration distance of different fixatives in heart tissue. A) A typical image of tissue before fixation; two dots were marked on a face of the tissue and were used for orientation, making sure that the tissue was immersed in the fixative with the marked face always facing up. B) A typical image of the marked face after fixation. C) The shrinkage or expansion of the heart tissue was calculated after exposure to different fixatives for $1,2,6$ or $10 \mathrm{~h}$ at $37^{\circ} \mathrm{C}$. $\mathrm{N}=4$; the change in the marked surface area ( $\Delta$ area) was calculated. The square root of the absolute value of the ratio of $\Delta$ area/area before fixation was then calculated as the shrinkage or expansion of the tissue; error bars represent standard deviation; positive values represent tissue expansion, whereas negative values represent tissue shrinkage. $D)$ The penetration distance was normalised to the tissue shrinkage or expansion of each individual sample. $n=4$; ${ }^{*} \mathrm{P}<0.001$, using two-way ANOVA followed by Bonferroni post-hoc tests. NS, not significant. 
were $1.7 \%, 0.9 \%$ and $6 \%$, respectively (Supplementary Table 3). The inter-observer coefficient of variation was $2.64 \%$ (Supplementary Table 4).

\section{Penetration rate of the fixatives in the heart tissue}

The penetration distance in the heart tissue increased along with the time of incubation for all the fixatives examined (Figure 2 ). The penetration distance of methanol in the heart tissue was significantly greater than that of ethanol $(\mathrm{P}<0.001$, Figures $2 \mathrm{~A}$ C) or formalin $(\mathrm{P}=0.003$, Figures $2 \mathrm{~A}, \mathrm{C})$. The penetration distance of ethanol was similar to that of formalin ( $\mathrm{P}=0.344$, Figure $2 \mathrm{C})$. The heart tissue shrank in ethanol or methanol whereas it expanded in formalin (Figures 1 A-C). The shrinkage caused by ethanol was similar to that caused by methanol $(\mathrm{P}=1.000)$. After normalising the penetration distance to the individual tissue's shrinkage or expansion, the penetration distance of methanol was significantly greater than that of ethanol or formalin $(\mathrm{P}<0.001$ for both, Figure 1D). The normalised penetration distance of ethanol was similar to that of formalin (Figure 1D).

The penetration distance in millimetres was plotted relative to the square root of the fixation time in hours (Figure 3) and the penetration coefficient $(\mathrm{K})$ was obtained. The mean penetration coefficients in the heart tissue were $2.609,1.994$ and 1.801 for methanol, formalin and ethanol, respectively (Figure 3). The penetration coefficient of methanol was significantly greater than that of ethanol or formalin ( $\mathrm{P}<0.001$ for both). The penetration coefficients of ethanol and formalin were similar $(\mathrm{P}=1.000)$.

\section{Penetration rate of the fixatives in the liver tissue}

The results obtained from the liver tissue were similar to those for the heart tissue. The penetration distance in the liver increased along with time of incubation for all the fixatives (Figure 4A). The penetration distance of methanol in the liver tissue was significantly greater than those of ethanol or formalin $(\mathrm{P}<0.001$ for both, Figure $4 \mathrm{~A})$. The penetration rate of ethanol was similar to that of formalin $(\mathrm{P}=1.000$, Figure 4A). The liver tissue shrank in all three fixatives. The shrinkage was significantly more in ethanol or methanol than in formalin $(\mathrm{P}<0.005$ for both, Figure 4B). The shrinkage caused by ethanol was similar to that by methanol $(\mathrm{P}=0.817$, Figure $4 \mathrm{~B})$. After normalising the penetration distance to the individual tissue's shrinkage or expansion, the penetration distance of methanol was significantly greater than those of ethanol or formalin $(\mathrm{P}<0.001$ for both, Figure 4C). The normalised penetration distance of ethanol was similar to that of formalin ( $\mathrm{P}=1.000$, Figure 1D).

The penetration distance in millimetres was plotted relative to the square root of the fixation time in hours (Figure 4D) and the penetration coefficient $(\mathrm{K})$ was obtained. The mean penetration coefficients in the liver tissue were $3.012,2.153$ and 2.113 for methanol, formalin and ethanol, respective-

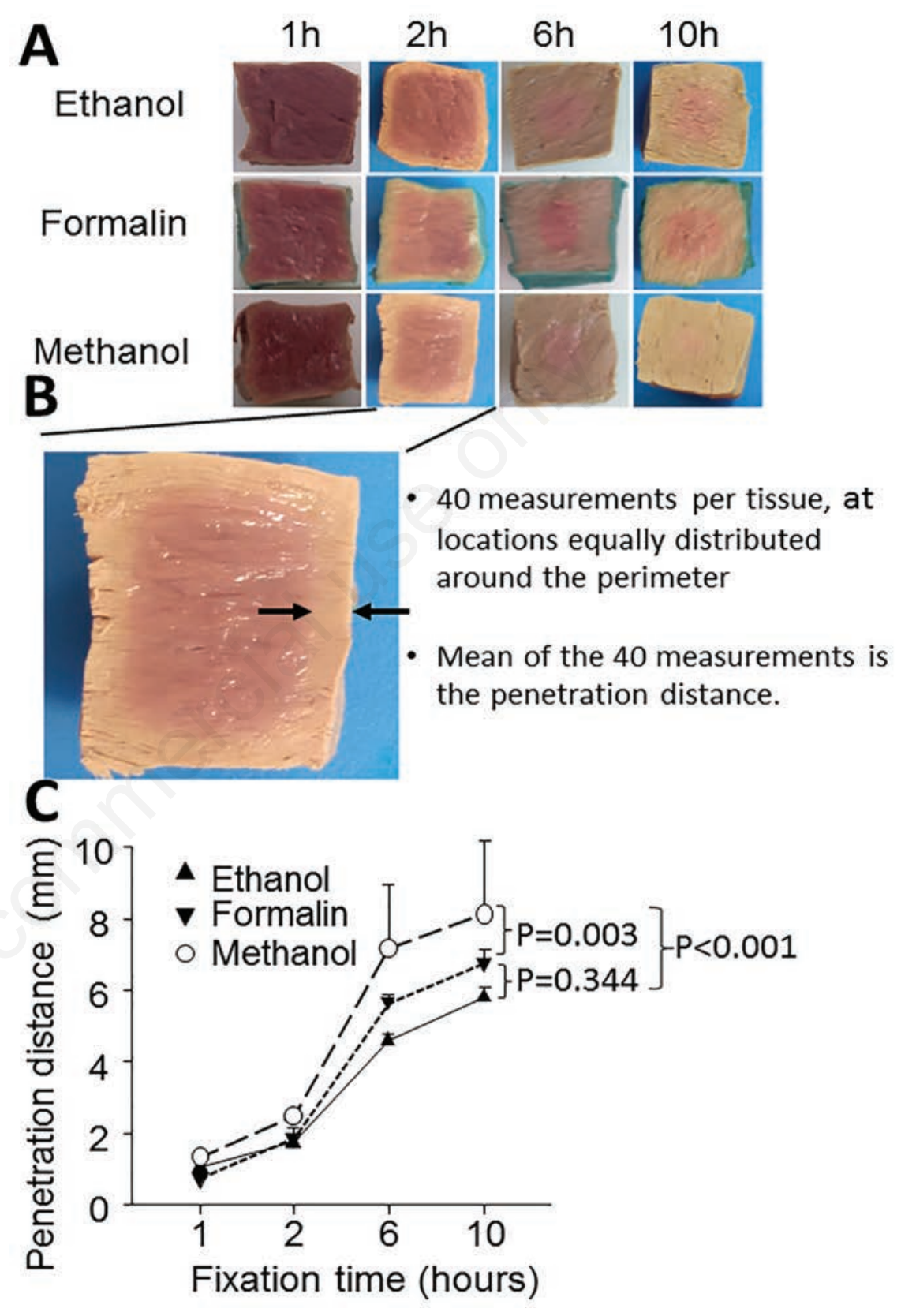

Figure 2. The penetration distance of different fixatives in heart tissue. A) Heart cubes (size: $2 \times 2 \times 2 \mathrm{~cm}^{3}$ ) were immersed in ethanol, methanol and $10 \%$ neutral-buffered formalin at $37^{\circ} \mathrm{C}$ with the marked face always facing up; the tissue was taken out of the fixatives at certain time points $(1,2,6$, or $10 \mathrm{~h})$ and cut into two halves along the middle plane parallel to the marked face and the newly cut face was then imaged with a digital camera. B) Representative image for the measurement of penetration distance; the penetration distance (the distance of discoloured region) was measured 10 times on each side using PhotoShop software and a total of $\mathbf{4 0}$ measurements were recorded for each tissue; the mean of the $\mathbf{4 0}$ measurements was calculated as the penetration distance of the fixative in this sample. C) Penetration distance over time for three fixatives; $n=4$; error bars represent standard deviation; the difference between fixatives was analysed using twoway ANOVA followed by Bonferroni post-hoc tests. 
ly (Figure 4D). The penetration coefficient of methanol was significantly greater than that of ethanol or formalin $(\mathrm{P}<0.001$ for both). The penetration coefficients of ethanol and formalin were similar $(\mathrm{P}=1.000)$.

\section{Discussion}

We report, for the first time, that the penetration rate of methanol was significantly greater than that of ethanol or formalin in bovine heart and liver tissues. This finding was unchanged after the correction for tissue shrinkage. The penetration rates of ethanol and $10 \%$ neutral-buffered formalin were similar in both tissue types. These results suggest that the assumption recently reported in a histology textbook that the penetration rate of methanol is similar to that of ethanol is incorrect. ${ }^{10}$

Many factors affect fixation, including temperature, tissue thickness, ratio of tissue to fixative volume, and fixation time. ${ }^{10}$ To appropriately study the difference in penetration rate of methanol and ethanol, we kept these variables consistent. In our study, the tissues were kept in a room in which

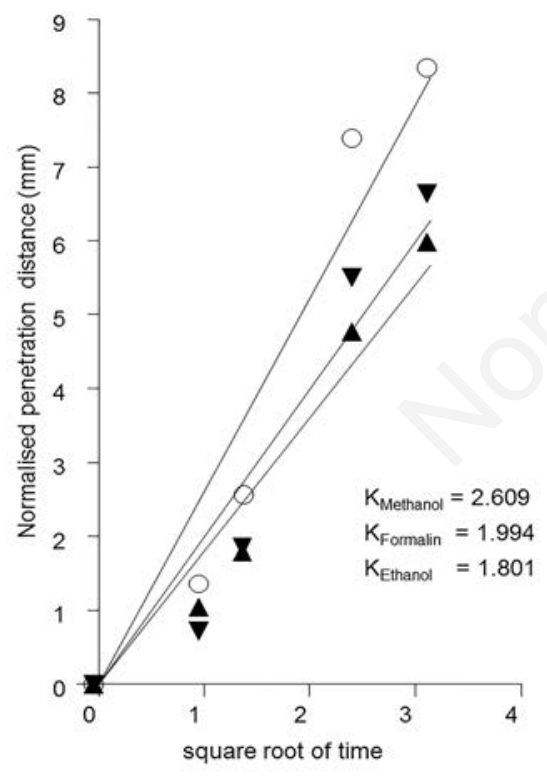

$\boldsymbol{\Delta}$ : ethanol; $\boldsymbol{\nabla}$ : formalin; $\mathrm{O}$ : methanol.

Figure 3. The depth of penetration of different fixatives into heart tissue relative to the square root of the fixation time. The penetration distance in millimetres was the mean of the measurements from four heart tissues. The $\mathbf{x}$-axis represents the square root of the fixation time in hours. For example, the value 3 on the $x$-axis represents $9 \mathrm{~h}$ of fixation. temperature was kept controlled at $37^{\circ} \mathrm{C}$. $2 \times 2 \times 2 \mathrm{~cm}^{3}$ tissue cubes and $40 \mathrm{~mL}$ of fixatives (tissue to fixative ratio of 1:5) were used in all experiments. We also examined penetration distances at different time points including immersion times of $1,2,6$ and $10 \mathrm{~h}$. We used 1:5 as the ratio of tissue to fixative volume. Researchers generally recommend a ratio of tissue to fixative volume around $1: 10$ or $1: 20 .^{7,10}$ However, this recommendation results from personal preferences without specific scientific evidence.
A

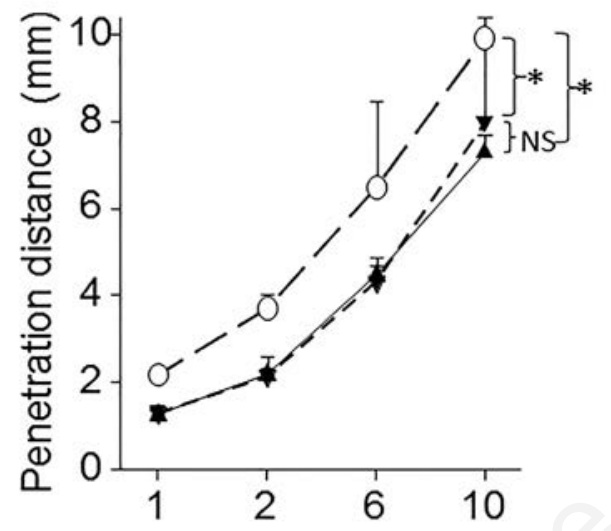

Fixation time (hours)
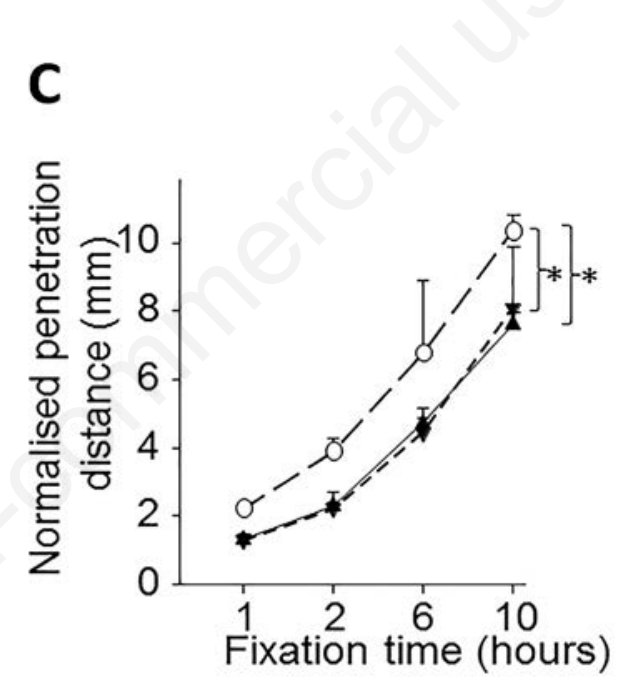

D
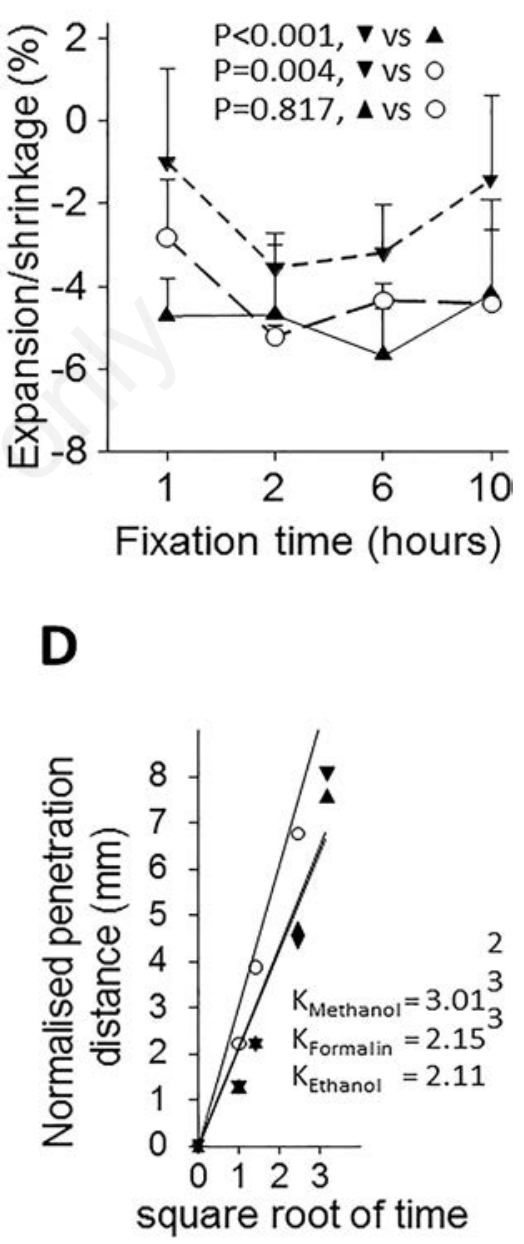

$\mathbf{\Delta}$ : ethanol; $\mathbf{v}$ : formalin; o : methanol.

Figure 4. The penetration distance of different fixative in liver tissue. A) Penetration distance of ethanol $(\Delta)$, formalin $(\nabla)$ and methanol $(\bigcirc)$ in the liver tissue; liver tissue cubes (size: $2 \times 2 \times 2 \mathrm{~cm}^{3}$ ) were immersed in fixative for $1,2,6$, or $10 \mathrm{~h}$ at $37^{\circ} \mathrm{C}$; the tissue was taken out of the fixative at certain time points and the penetration distance was then measured using PhotoShop software; $n=4$; error bars represent standard deviation; ${ }^{*} \mathbf{P}<\mathbf{0 . 0 0 1}$, using two-way ANOVA followed by Bonferroni post-hoc tests; NS, not significant. B) The shrinkage or expansion of the liver tissue. The shrinkage or expansion was calculated after exposure to the fixatives for $1,2,6$ or $10 \mathrm{~h}$ at $37^{\circ} \mathrm{C} ; \mathrm{n}=4$; the change in the marked surface area $(\Delta$ area) was calculated; the square root of the absolute value of the ratio of $\Delta$ area/area before fixation was then calculated as the shrinkage or expansion of the tissue; error bars represent standard deviation; positive values represent tissue expansion, whereas negative values represent tissue shrinkage. C) The penetration distance in the liver tissue was normalised to the tissue shrinkage or expansion of each individual sample; $\mathrm{n}=4$; error bars represent standard deviation; ${ }^{*} \mathrm{P}<0.001$, using two-way ANOVA followed by Bonferroni post-hoc tests. D) Depth of penetration of fixatives into the heart tissue relative to the square root of the fixation time; the penetration distance in millimetres was the mean of the measurements from four liver tissues; the $x$-axis represents the square root of the fixation time in hours. 
Williams et al. have reported the effects of fixation on immunohistochemistry procedures and concluded that there were no differences in the results obtained after fixing human tonsils with formalin at tissue to fixative volume ratios between 1:1 and 1:20. ${ }^{13}$ Buesa and Peshkov investigated the tissue to fixative volume required for quality fixation using formalin. They reported that the fixation quality was not effected by different tissue to fixative volume ratios such as $1: 1,1: 2,1: 5$, and 1:10. ${ }^{14}$ Therefore, we believe the tissue to fixative volume ratio of 1:5 we used was appropriate.

When we immersed the tissue cubes into the different fixatives, we did not place a mesh on the bottom of the containers. It has been reported that a plastic mesh on the bottom of fixation containers could guarantee uniform access of the fixative to all surfaces of the tissue pieces during fixation. ${ }^{7}$ However, we marked the top face with tissue ink, and the marked face always pointed up during the whole experiment. The bottom face of the tissue in our experiment had limited fixation due to limited access to the fixatives. However, this did not affect our measurement of penetration distance. This is because the fixative penetrated freely from the other sides of the cubes. When we cut the tissue open for penetration distance measurement, we always cut the tissue along the middle plane parallel to the bottom face.

We used PhotoShop to measure the penetration distance of fixatives, instead of using less accurate methods such as using micrometre eyepiece. ${ }^{11}$ In addition, when we measured the penetration distance, we performed measurements 40 times at locations equally distributed around the perimeter of the cut face. Our method is in contrast to previous reports such as those by Cabrera and colleagues where only 4 measurements were used to calculate the mean penetration distance. ${ }^{7}$ We demonstrated that the method we used had very good reproducibility. The intra-observer coefficient of variation of the penetration distance ranged from $0.9-6.0 \%$ and inter-observer coefficient of variation was $2.6 \%$.

Previous reports on the penetration rate of fixatives are scarce and our understanding on this topic is mainly based on a few studies performed many years ago. Dempster reported that penetration of the fixative osmium tetroxide was proportional to its fixation time. ${ }^{8}$ Medawar, ${ }^{12}$ Dempster, ${ }^{8}$ and Start et al. ${ }^{1}$ reported that the distance of penetration of various fixatives was a linear function of the square root of fixation time, i.e. $\mathrm{d}=\mathrm{K} V_{\mathrm{t}}$, where " $\mathrm{d}$ " is penetration distance in millimetres, " $\mathrm{K}$ " is penetration coefficient, and " $\mathrm{t}$ " is time in hours. This relationship, which has been referred to as the law of diffusion $(\mathrm{d}=\mathrm{K} \mathrm{V})$, between penetration distance and the square root of fixation time was observed in various tissues including blood plasma coagulum, ${ }^{12}$ rabbit liver $^{8}$ and human spleen. ${ }^{1}$

Our result confirmed the law of diffusion in both bovine heart and liver tissues. The penetration coefficient of methanol was higher than that of ethanol in both the heart and liver tissues providing the first evidence that the penetration rate of methanol is higher than ethanol. In addition, the penetration coefficient of methanol or ethanol in the liver was greater than that in the heart. This suggests that the penetration rate is tissue specific. It has been reported that the penetration of a fixative into muscles was more rapid than into the brain. ${ }^{8}$ The difference in penetration rate between the liver and heart tissues reported in our study may be due to the fact the liver tissue is less dense compared to the heart tissue and this may facilitate diffusion of fixatives.

The mean penetration coefficients of ethanol in bovine heart and liver (1.801 and 2.113 ) in our study were slightly higher than the previously reported value of 1.714 in blood plasma coagulum. ${ }^{12}$ This may be due to the higher room temperature used in our study compared to the previous report by Medawar. ${ }^{12}$ Dempster ${ }^{8}$ reported the mean penetration coefficient of $95 \%$ ethanol at room temperature $\left(21-25^{\circ} \mathrm{C}\right)$ in rabbit livers was approximately 1.6. The difference between Dempster's report and our observation may be due to differences in the concentration of ethanol ( $95 \%$ vs $100 \%)$, temperature and tissue source (rabbit vs bovine).

The reported formalin $(10 \%)$ penetration coefficient has also varied. Start et al. ${ }^{1}$ reported that the mean penetration coefficient for formalin was 0.55 in human spleens at $20^{\circ} \mathrm{C}$. Chesnick et al..$^{15}$ reported that it was 0.47 at $22^{\circ} \mathrm{C}$ and 1.1 at $42^{\circ} \mathrm{C}$ in porcine liver. ${ }^{15}$ The mean penetration coefficient of formalin was 1.994 and 2.153 in bovine heart and liver tissue, respectively, in our study. The different reported results may reflect differences in experimental temperature and structure of the various tissues used.

Fixatives can cause tissue expansion or shrinkage. ${ }^{10}$ To study the effect of fixatives on tissue expansion or shrinkage, we marked one face (top face) of the tissue cubes, and measure its size before and after fixation. This method of measuring the area of a tissue face had good reproducibility, with the intra-observer coefficient of variation ranging from $0.7-1.2 \%$ and inter- observer coefficient of variation being $1.4 \%$. We found that methanol and ethanol shrank both heart and liver tissues. This is consistent with previous reports. ${ }^{7,10}$ Our results also showed, for the first time, that the degree of shrinkage caused by methanol and ethanol was similar. By contrast, we found that formalin expanded the heart tissue but shrank the liver tissue. The tissuespecific effects of formalin have been reported in previous studies. It has been reported that after porcine forelimb tissue was immersed in formalin it expanded, but that fat tissue shrank, and bone tissue was not changed. ${ }^{16}$

In summary, this study suggests that the penetration rate of methanol is significantly greater than that of ethanol and formalin in both bovine heart and liver tissues. Our results suggest that the assumption that the penetration rate of ethanol is similar to that of methanol is incorrect. ${ }^{10}$

\section{References}

1. Start RD, Layton CM, Cross SS, Smith JH. Reassessment of the rate of fixative diffusion. J Clin Pathol 1992;45:1120-1.

2. Srinivasan M, Sedmak D, Jewell S. Effect of fixatives and tissue processing on the content and integrity of nucleic acids. Am J Pathol 2002;161:1961-71.

3. Werner M, Chott A, Fabiano A, Battifora H. Effect of formalin tissue fixation and processing on immunohistochemistry. Am J Surg Pathol 2000;24: 1016-9.

4. Alshammari MA, Alshammari TK, Laezza F. Improved methods for fluorescence microscopy detection of macromolecules at the axon initial segment. Front Cell Neurosci 2016;10:5.

5. Harrison PT. An ethanol-acetic acid-formol saline fixative for routine use with special application to the fixation of non-perfused rat lung. Lab Anim 1984; 18:325-31.

6. Mathieson W, Marcon N, Antunes L, Ashford DA, Betsou F, Frasquilho SG, et al. A critical evaluation of the PAXgene tissue fixation system: Morphology, immunohistochemistry, molecular biology, and proteomics. Am J Clin Pathol 2016;146:25-40.

7. Cabrera NC, Espinoza JR, VargasJentzsch P, Sandoval P, Ramos LA, Aponte PM. Alcohol-based solutions for bovine testicular tissue fixation. J Vet Diagn Invest 2017;29:91-9.

8. Dempster WT. Rates of penetration of fixing fluids. Am J Anat 1960;107:59-72.

9. Staff S, Kujala P, Karhu R, Rokman A, 
Ilvesaro J, Kares S, et al. Preservation of nucleic acids and tissue morphology in paraffin-embedded clinical samples: comparison of five molecular fixatives. J Clin Pathol 2013;66:807-10.

10. Carson FL, Hladik C. Histotechnology. 4th ed. Chicago: American Society for Clinical Pathology; 2014.

11. Underhill BML. V.-The rate of penetration of fixatives. $T$ Roy Micr 1932;52:113-20.
12. Medawar PB. III.-The rate of penetration of fixatives. T Roy Micr 1941; 61:46-57.

13. Williams JH, Mepham BL, Wright DH. Tissue preparation for immunocytochemistry. J Clin Pathol 1997;50:422-8.

14. Buesa RJ, Peshkov MV. How much formalin is enough to fix tissues? Ann Diagn Pathol 2012;16:202-9.

15. Chesnick IE, Mason JT, O'Leary TJ, Fowler CB. Elevated pressure improves the rate of formalin penetration while preserving tissue morphology. J Cancer 2010;1:178-83.

16. Docquier PL, Paul L, Cartiaux O, Lecouvet F, Dufrane D, Delloye C, et al. Formalin fixation could interfere with the clinical assessment of the tumorfree margin in tumor surgery: magnetic resonance imaging-based study. Oncology 2010;78:115-24. 